\title{
The potential of microelectrode arrays and microelectronics for biomedical research and diagnostics
}

\section{Review Article}

Author(s):

Jones, lan L.; Livi, Paolo; Lewandowska, Marta K.; Fiscella, Michele; Roscic, Branka; Hierlemann, Andreas

Publication date:

2011-03

Permanent link:

https://doi.org/10.3929/ethz-b-000158716

Rights / license:

In Copyright - Non-Commercial Use Permitted

Originally published in:

Analytical and Bioanalytical Chemistry 399(7), https://doi.org/10.1007/s00216-010-3968-1 


\title{
The potential of microelectrode arrays and microelectronics for biomedical research and diagnostics
}

\author{
Ian L. Jones • Paolo Livi • Marta K. Lewandowska • \\ Michele Fiscella • Branka Roscic • Andreas Hierlemann
}

Received: 20 May 2010 / Accepted: 23 June 2010/Published online: 31 July 2010

(C) Springer-Verlag 2010

\begin{abstract}
Planar microelectrode arrays (MEAs) are devices that can be used in biomedical and basic in vitro research to provide extracellular electrophysiological information about biological systems at high spatial and temporal resolution. Complementary metal oxide semiconductor (CMOS) is a technology with which MEAs can be produced on a microscale featuring high spatial resolution and excellent signal-to-noise characteristics. CMOS MEAs are specialized for the analysis of complete electrogenic cellular networks at the cellular or subcellular level in dissociated cultures, organotypic cultures, and acute tissue slices; they can also function as biosensors to detect biochemical events. Models of disease or the response of cellular networks to pharmacological compounds can be studied in vitro, allowing one to investigate pathologies, such as cardiac arrhythmias, memory impairment due to Alzheimer's disease, or vision impairment caused by ganglion cell degeneration in the retina.
\end{abstract}

Keywords Biochips · Biosensors · Electroanalytical methods $\cdot$ Microelectrode array $\cdot \mathrm{CMOS}$

\section{Introduction}

The growing importance and increasing sophistication of biomedical technology is leading a trend toward the

Published in the special issue Analytical Challenges in Nanomedicine with Guest Editors Boris Mizaikoff, Douglas C. Eaton, and Christine Kranz.

I. L. Jones $(\varangle) \cdot$ P. Livi $\cdot$ M. K. Lewandowska $\cdot$ M. Fiscella

B. Roscic $\cdot$ A. Hierlemann

Department Biosystems Science and Engineering (BSSE),

Eidgenössische Technische Hochschule Zürich (ETHZ),

Mattenstrasse 26,

4058 Basel, Switzerland

e-mail: ian.jones@bsse.ethz.ch diagnosis and study of disease on the microscale. While well-established methods in electrophysiology, such as the electrocardiogram (ECG) and electroencephalogram (EEG), are regularly used to monitor the health of patients and in models of disease, there is an increasing need for methods that measure the electrical activity and biochemistry of tissue in ever greater detail. In recent years, rapid advances in complementary metal oxide semiconductor (CMOS) technology have made construction of circuits on a microscale possible. This technology provides information about electrophysiological and biochemical events that occur at the cellular or even molecular level can be obtained.

Planar CMOS microelectrode arrays (MEAs) are the focus of this review, as they are ideally suited for the study of complex cellular networks and sensitive molecule detection in vitro. Such technology is crucial in applications such as pharmacological drug screening and highthroughput systems for electrophysiological testing. Furthermore, such a system is invaluable in basic research, where a complex in vitro system can be used to examine network behavior in cell populations. CMOS technology has the advantage of being robust, straightforward to use, and it can yield immense amounts of data from biological systems in vitro.

\section{Electrophysiology and multielectrode arrays}

The vital processes in the human body are regulated by the activity of electrogenic cells that are connected in networks. Some examples of such activity are: information processing and neurotransmission between cells in the brain; lightdriven responses transmitted through retinal ganglion cells; the synchronized beating of cardiomyocytes in the heart. 
Electrogenic cells essentially behave as signal receivers and generators, conveying information from one part of the body or organ to another via intercellular transmission. Cellular signals originate in the following manner: each cell has a concentration gradient of ions across its membrane, which means that there is an electrochemical potential difference across the membrane, called the membrane potential. A transitory alteration of the membrane potential is caused by the acceptance and release of ions across the cell membrane, causing the number of ions in the extracellular environment - and therefore the local charge density-to change. When the membrane potential becomes more positive and reaches a threshold value greater than its normal resting membrane potential, the cell depolarizes rapidly, causing an action potential, followed by a repolarization back to baseline.

In the field of electrophysiology, there are two principal methods by which the electrical activity of electrogenic cells can be measured: intracellular and extracellular. Intracellular electrophysiologic activity of the cell is detected by the patch clamp device; this is a device, which features an electrode that is inserted into the cell, and a second electrode that, e.g., can be sealed to a small region of the cell and measures the flux of ions across the membrane $[1,2]$. There are multiple patch clamp configurations (single ion channel, whole cell, etc.) and there are also robotically controlled multi-patch clamp setups that can patch several cells simultaneously [3]. External microtransducers are used to measure the extracellular electrophysiological activity, which occurs outside of the cell membrane [4-14]. Microtransducers measure the concentration of ions in their vicinity, which changes according to the activity of the cell. Upon the occurrence of electrical activity of the cell, a so-called action potential, ions, mostly sodium and potassium ions, travel across the cell membrane in and out of the cell. These moving ions generate an electric field, which can directly influence the open-gate region of a field-effect transistor $[15,16]$, or which can be recorded by means of metal microelectrodes [17-21], as shown in the schematic in Fig. 1. This enables the measurement of the extracellular voltage or current that is produced by the cell when it undergoes an action potential. Additional methods that indirectly indicate the voltage change of a cell include optical measurements using voltage-sensitive or fluorescent dyes [22-24].

Measurement of the simultaneous and interdependent activity of electrogenic cells is crucial, since the cells respond to a complex set of environmental and contextual stimuli. It is therefore desirable to have the capability to measure the behavior of many cells simultaneously to reveal network characteristics and, at the same time, to have the possibility to conduct measurements at high spatial resolution, ideally subcellular resolution, to reveal details of

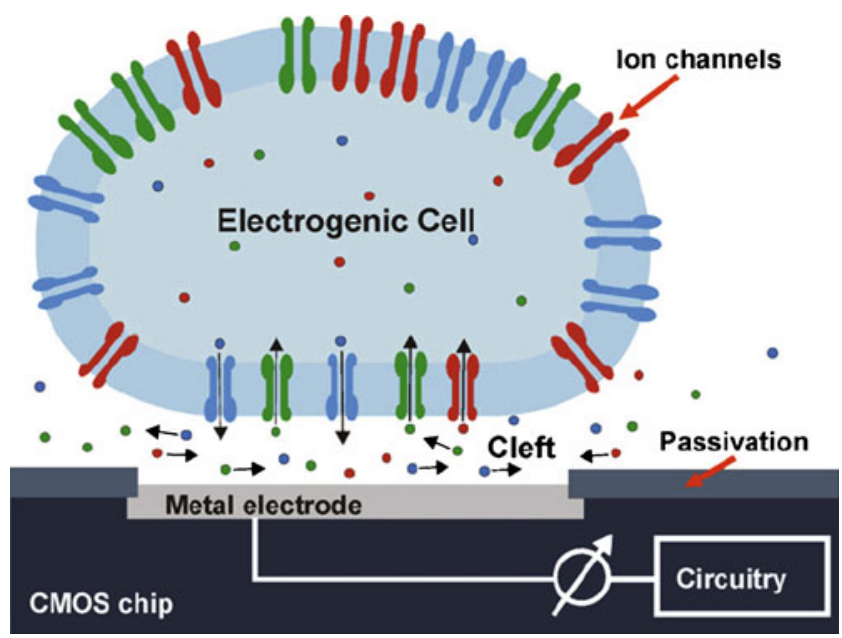

Fig. 1 Schematic of a cell in close proximity to an example planar microtransducer (open-gate transistor/electrode). The displayed cell features ion channels; ions that move through these channels generate an electric field or voltage change that is recorded by the microtransducer [25]

cellular signaling. The previously mentioned techniques of directly measuring the electrophysiological signal are limited in this respect; to overcome these limitations, a multielectrode array (MEA) or multitransducer array may be a more suitable device. An MEA is a two-dimensional grid of biocompatible microtransducers embedded in an insulating surface, such that each microtransducer is electrically independent of its neighbors. Each transducer is connected to a differential amplifier, which measures voltage or current with respect to a common reference. Thus, an MEA provides a regular spatial sampling of the voltage or current signals across a two-dimensional plane. A high-density (HD) MEA is a two-dimensional array of densely packed transducers that can measure the responses from many cells simultaneously; depending on the density and capabilities of the array and the cell density, signals can be acquired simultaneously from all cells within a culture or slice. Finally, it should be noted that in contrast to methods such as patch clamping, cells can be maintained for up to several months so that long-term studies can be conducted.

While it is useful to have the ability to record the activity of cells, there are some instances in which intrinsic activity alone does not provide sufficient information; stimulation of the slice or culture is necessary to study certain aspects of cellular network behavior or activity; for example, phenomena such as memory formation in brain tissue or pacemaking in the heart often require an external stimulus. To provide this capability, some MEA devices have bidirectional functionality, meaning that they can not only record activity, but can deliver either a current or voltage stimulus, thereby eliciting a response in a population of electrogenic cells. These devices can be used to stimulate a single cell or small number of cells with one or more 
electrodes. The subsequent cell population response can then be recorded with the non-stimulating electrodes [2629]. Likewise, chemical concentrations and gradients provide a wealth of information about cell processes and communication. Two- or three-electrode measurements can be made by using MEAs, which detect the presence of electroactive or non-electroactive neurotransmitters or other relevant ions or molecules [30].

The MEA as a device for electrophysiological recording has been in existence for several decades and has undergone several improvements throughout this time. One of the early planar MEAs was described in a 1972 publication by Thomas et al. (Fig. 2), who aimed to record electrical activity from cells using a noninvasive device, allowing them to address questions involving plasticity and electrical interaction among cells. The array comprised 15 gold electrodes with $100 \mu \mathrm{m}$ spacing; experiments with dorsal root ganglion cells were carried out [31]. A similar MEA device was published 5 years later by Gross et al. [32], while the first successful recordings from dissociated neurons were reported by Pine in 1980 [33]; in the latter case, an MEA with two parallel rows of 16 planar gold

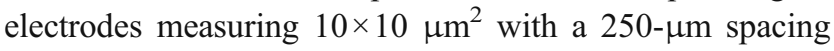
was developed.

An alternative approach to the use of metal electrodes was presented in 1970 by Bergveld [35]. He developed an open-gate field-effect transistor (FET) in order to measure ionic concentrations in a solution. This device, called ISFET (ion-sensitive FET), relies on the same working principle as a normal metal oxide semiconductor FET
(MOSFET); however, the current flow is regulated (gated) by a reference electrode and the concentration of ions in the solution (Fig. 2) instead of a metal gate electrode (MOSFET). Bergveld successfully used ISFETs to measure ions fluxes around a nerve [36]. Similarly, in 1981 Jobling et al. reported a nine-electrode MEA in which the gates of a FET on a silicon chip were used as electrodes [37]. The firing neurons were located close to the exposed gate of the transistor, which behaved as a sensor; changes in the extracellular potential directly modulated the current flowing through the transistor. Successful recordings from hippocampal slices with high signal-to-noise ratio were demonstrated.

\section{CMOS technology}

There are a variety of MEAs available for electrophysiological measurements. In most commercially available systems (e.g., Multi Channel Systems GmbH, Germany; Panasonic Inc., Japan; Plexon Inc., USA), passive metal electrode arrays are employed. These devices usually consist of 64 electrodes and are aligned on a glass or silicon substrate with externally situated signal recording and filtering components. However, transducers can also be realized by using standard integrated circuit or complementary metal oxide semiconductor (CMOS) technology. There are three primary advantages to using this technology: (1) connectivity: onchip multiplexing means that many electrodes can be addressed, allowing for measurements at high spatiotempo-
Fig. 2 Top: diagrammatic cross section of microelectrode array forming the floor of a culture chamber, reprinted from [31]. Bottom: schematic representation of a standard metal oxide semiconductor field-effect transistor, MOSFET (a) and an ionsensitive field-effect transistor, ISFET (b). Reprinted from [34]

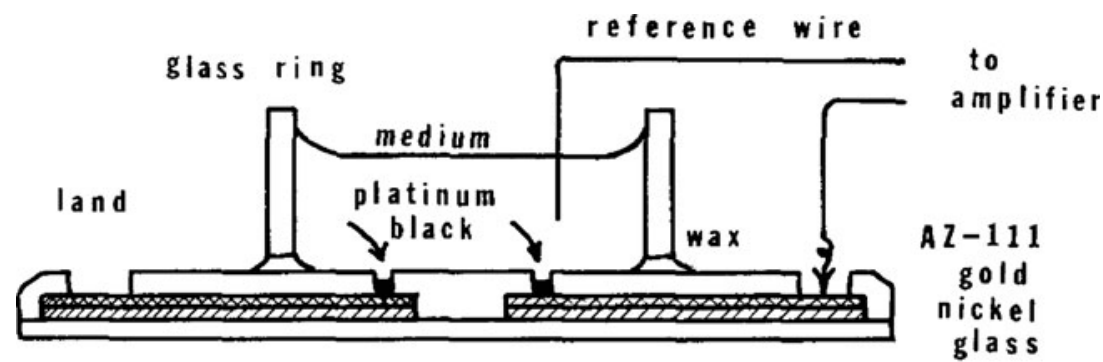

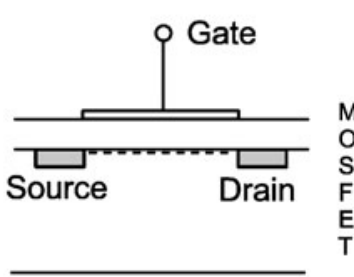

(a)

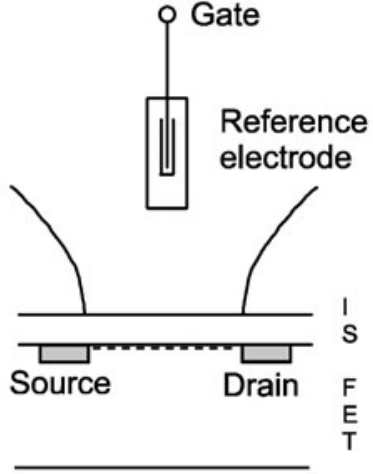

(b) 
a

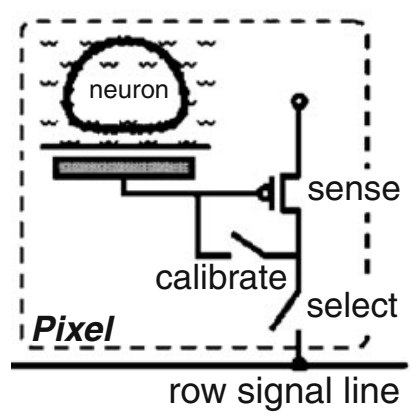

b

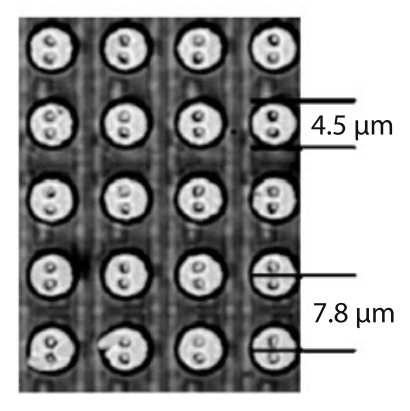

C

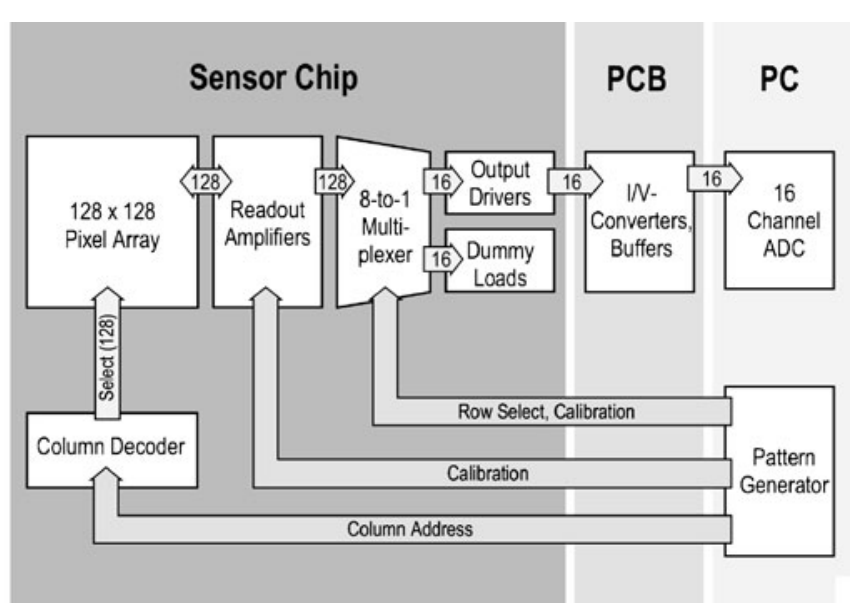

Fig. 3 High-density sensor array with 16,384 pixels based on open-gate field effect transistors on a $1 \times 1 \mathrm{~mm}^{2}$ area. a Pixel schematic. b Zoom-in on sensor array. c System architecture [40]

ral resolution; (2) signal quality: signal processing circuitry can be located directly below the electrode, facilitating the detection of weak signals through immediate signal conditioning and digitization; (3) ease of use: many functions can be implemented via user-friendly software that communicates with on-chip logic units through a digital interface. Moreover, the use of CMOS technology allows for the realization of small system chips with large numbers of electrodes; arrays of up to 16,384 electrodes on $6 \mathrm{~mm}$ by $6 \mathrm{~mm}$ chips have been reported [38-42].

High-density microelectrode arrays realized in standard CMOS technology offer the potential to perform recordings at single-cell or even subcellular resolution [42]. When designing such systems, a compromise between noise performance and spatial resolution must be made. To achieve high spatial resolution, small electrodes are preferred; unfortunately, thermal noise is inversely correlated to electrode size. A further impediment is that, because of spatial constraints, filters and amplifiers with large noise-reducing capacitors cannot be used.

Another issue that must be taken into account with CMOS MEAs is the packaging of the chip. The standard metal used in CMOS technology is aluminum, which is a neurotoxin, so it must not come into contact with cell cultures. Moreover, electronic circuits are corroded and destroyed by aqueous salt solutions, so the chips themselves need to be protected from the very things they measure.

A device relying on FET transducers in CMOS technology was developed at the Max Planck Institute for Biochemistry, Germany, by the group of P. Fromherz [40, 43]; this CMOS MEA featured 16,384 open-gate FETs at a pitch of $7.8 \mu \mathrm{m}\left(16,000\right.$ sensors $\left./ \mathrm{mm}^{2}\right)$, as shown in Fig. 3.

The array was composed of $128 \times 128$ pixels, each comprising three transistors for voltage sensing, pixel selection, and calibration to compensate for transistor mismatch and leakage. The sensor chip also incorporates a column decoder to control each pixel, a readout amplifier for each of the 128 rows, and an 8-to-1 multiplexer to drive the outputs of the amplifiers. The generated output currents are transferred to a set of off-chip current-to-voltage converters whose output is digitized by 16 analog-todigital converters (ADCs). In order to maintain high spatial resolution, a small pixel size must be balanced against the need for circuits that are sufficiently large to reduce signal noise, which can range from 70 to $250 \mu \mathrm{V}_{\mathrm{RMS}}[38,40]$.

CMOS-based devices relying on planar metal electrodes also have been devised: an MEA with integrated multiplexers and in-pixel amplifiers that can simultaneously record from 4,096 aluminum or gold electrodes was presented by Berdondini et al.; the electrodes were constructed with a $40-\mu \mathrm{m}$ pitch, which is equivalent to approximately 500 electrodes $/ \mathrm{mm}^{2}$ [39, 44-46]. The system is based on the active pixel sensor (APS) concept, originally designed for image sensors; it is composed of a $64 \times 64$ pixel or electrode array with a pixel area of $40 \times$ $40 \mu^{2}$. Data streams can be collected from the whole array at $7.7 \mathrm{kHz}$, but it is also possible to select specific regions of interest and to record at higher temporal resolutions of up to $120 \mathrm{kHz}$ [44, 45]. In this design, noise-optimized and spatially optimized operational transconductance amplifiers consisting of only five transistors have been employed. The noise of the system is approximately $10-20 \mu \mathrm{V}_{\text {PP. }}$ The chip also includes filtering circuits, high-speed addressing logic, and a field-programmable gate-array interface, which provides dynamic analog-to-digital (AD) conversion as well. The system is linked to a computer running custom software for data acquisition and analysis. A chip micrograph, the overall system overview, and the amplifier schematics are shown in Fig. 4.

A CMOS chip with stimulation capabilities was presented by the group of Kovacs at Stanford [47], using an 


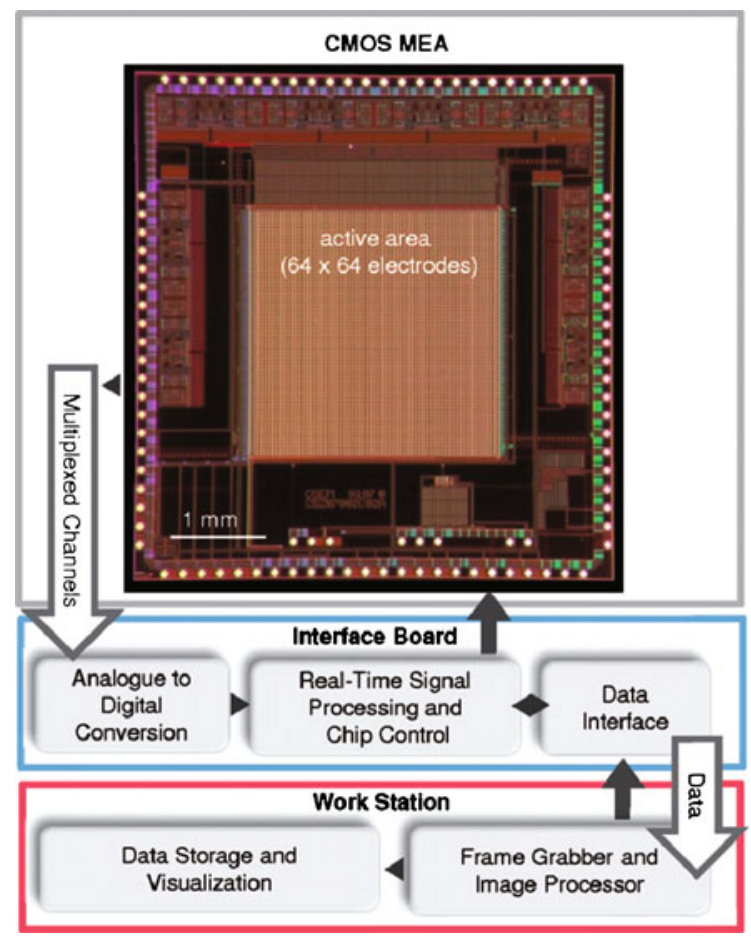

(A)
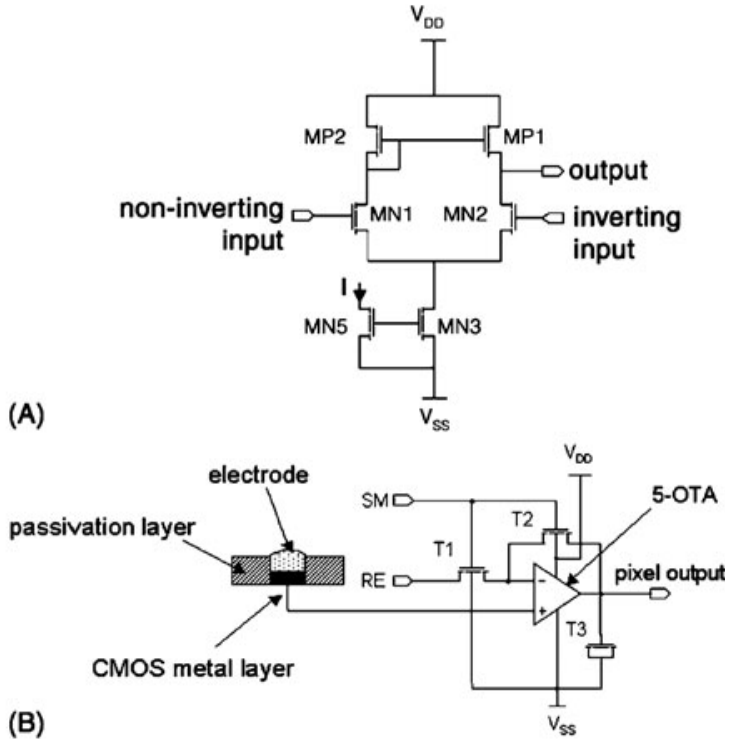

Fig. 4 Left: chip micrograph and system overview of the high resolution CMOS-MEA presented in [46]. Right: a schematic of the in-pixel fivetransistor preamplifier. b Complete schematic of the in-pixel amplifier (OTA, operational transconductance amplifier). Reprinted from [39]

integrated stimulation electrode outside the recording electrode array. CMOS-based needle arrays featuring stimulation and recording electrodes for insertion into living tissue were reported by Wise and Najafi [5, 48, 49]; these devices for in vivo applications feature multiple electrodes per needle shank and integrated circuitry units, including radio frequency transmission, on the chip base. Several of them can be assembled in 3D electrode arrays.

Monolithic bidirectional CMOS MEAs, which have stimulation and recording capabilities, have been published by Heer et al. [25, 50, 51] and Frey et al. [41, 42]. Both system chips employ planar metal electrodes and are used directly as a substrate for cell and slice culturing or for accommodating acute tissue slices; they include circuitry for stimulation, immediate cell signal modulation, analogto-digital conversion, and a digital interface, thus allowing a rapid interaction approaching real-time functionality.

The system of Heer et al. includes 128 electrodes and a reference electrode. Each pixel is adjacent to an electrode and contains a band-pass filter, a mode storage unit, a buffer for stimulation; the pixel pitch is $250 \mu \mathrm{m}$. The chip also includes a digital control unit in order to provide multiplexing, the electrode selection for stimulation, the resetting of single electrodes and the $\mathrm{AD}$ converters, and the interface to the external world. A temperature sensor is included to ensure that electrogenic cells are at the correct temperature. The close proximity of filters and buffers to each electrode offers several advantages: (1) reduced interference-induced noise at connection lines; (2) a highpass filter, which reduces offset and drift; (3) a low-pass filter that limits noise bandwidth and prevents aliasing in the multiplexing and analog-to-digital conversion stages. The measured noise of the in-pixel circuitry is $11.7 \mu \mathrm{V}_{\mathrm{RMS}}$, and the total power dissipated is $120 \mathrm{~mW}$. Any arbitrary stimulation configuration can be applied to any subset of electrodes. Moreover, every recording channel can be reset to its initial state in order to suppress stimulation artifacts.

Figure 5 shows the implementation of Frey et al.'s design that achieves high signal-to-noise ratios and high spatial resolution. The system features 11,011 electrodes and an analog switching matrix, located directly underneath the electrode array. This switching scheme is compact, has low-noise characteristics, and allows the routing of an arbitrarily selectable subset of electrodes to 126 readout channels.

In this system, electrodes are small, with a diameter of $7 \mu \mathrm{m}$ and a pitch of $17 \mu \mathrm{m}$. Signal modification circuitry has therefore to be placed outside of the array, where space constraints are no longer a concern. The array area is $2 \times$ $1.75 \mathrm{~mm}^{2}$, which yields a density of 3,150 electrodes $/ \mathrm{mm}^{2}$. The electrode selection can be adapted to the biological sample of interest, while the integrated circuitry can be optimized for noise, which is as low as $4 \mu V_{\text {RMS }}$. Moreover, the large redundancy of the data obtained from such a multi-data point recording (each neuronal activity is simultaneously recorded by many electrodes because of the 

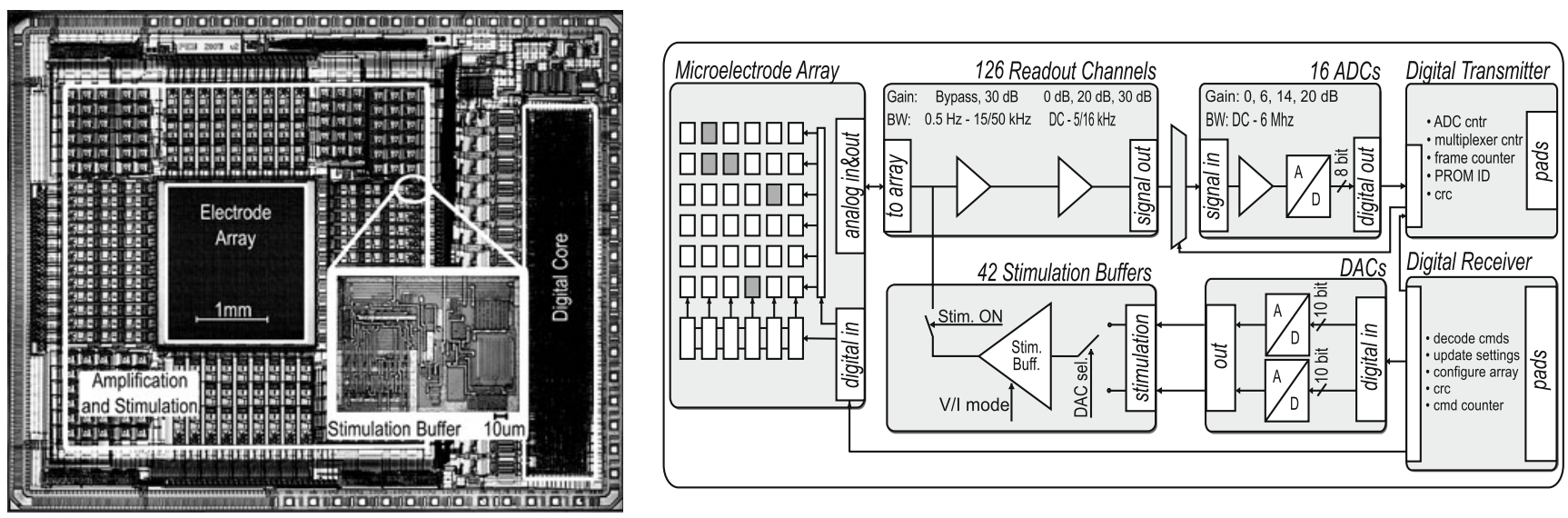

Fig. 5 Left: 11,011-electrode CMOS chip micrograph. The electrode array is in the center, surrounded by the analog circuitry for filtering, amplification, and stimulation; on the right side sits the digital interface. Right: block diagram of the on-chip circuitry. Reprinted from [52]

tight spacing) can be used for spike sorting software algorithms that separate action potentials based on shape and occurrence pattern. This system makes it possible to obtain a high spatially and temporally resolved reconstruction of the extracellular activity of a cell or cell population.

A block diagram of the system is depicted in the right side of Fig. 5. The recorded signals are amplified and filtered in three stages, each of which has programmable gain. After the second stage, signals are multiplexed, sampled, and digitized; finally, the data are transferred by a digital interface to off-chip computational machines. The same interface also controls the array configuration and stimulation mode settings. Stimulation is performed by integrated buffers, which have both voltage and current modes for stimulation delivery at an arbitrary electrode subset [52].

Both the Heer and Frey systems were fabricated with standard 0.6- $\mu \mathrm{m}$ CMOS technology; special post-CMOS processing and packaging for operation under physiological conditions were subsequently applied. Electrodes were covered by biocompatible platinum and a four-layer passivation stack of alternating silicon dioxide and silicon nitride was deposited on the chip and opened at the site of the electrodes [50]. Finally, the chips were mounted on a custom-designed printed circuit board (PCB) for long-term culturing.

\section{Biological preparation methodology}

The most common biological preparations studied using MEAs are divided into two main categories: acute tissue slices, which are recorded immediately after they are removed from the animal; and cell cultures, which can be further divided into dissociated cell cultures and organotypic tissue cultures. Dissociated cultures are cells whose relative in vivo positions are no longer preserved, whereas organotypic tissue cultures are slices that are maintained in vitro over a period of time. These variations encompass the spectrum from short-term, functionally preserved tissue slices to long-term organotypic or cell culture preparations.

Several parameters must be taken into consideration when using MEAs as a tool for recording from cells. Most importantly, the neuron-electrode interface must be optimized so that tight coupling between the cells and electrodes is established, maximizing the signal-to-noise ratio. Acute slices, for example, have a layer of dead cells that are damaged during the cutting of the tissue, which introduces a degree of isolation between the active cells and the electrodes. To promote the adhesion of cells or the tissue mass, MEAs are coated with cell-adhesion substrates such as poly-D-lysine, laminin, collagen, and fibronectin [53, 54]. Successful tactics for making recordings from tissue sections utilize, for example, cellulose nitrate or coagulated plasma/thrombin clots to stabilize the slices on the MEA surface [55].

Acute tissue slices, including brain tissue and retina, preserve the structural and functional relationships between groups of cells because there is no opportunity for the cells to alter their relative formation from the initial in vivo state. Acute slices are cut at a thickness ranging from approximately 100 to $400 \mu \mathrm{m}$, although the maximum distance away from an electrode that a signal can obtained is approximately $100 \mu \mathrm{m}$ [56]. Some high-density MEAs are capable of recording from as far away as $100 \mu \mathrm{m}$, but the signal-to-noise ratio drops off rapidly with distance [57]. Brain slices prepared from mice and rats at postnatal day 6 12 were reported to exhibit optimal cell health and maintained neurogenesis [58].

The major limitation of acute slice preparations is the duration of viability, which is typically up to several hours 
following slice preparation. Furthermore, a perfusion system is necessary for the delivery of carbogen gas $\left(95 \% \mathrm{O}_{2} / 5 \% \mathrm{CO}_{2}\right)$ and nutrients, which are required to sustain the physiologic processes of the cells. It must also be considered that inputs originating from diffuse neuromodulatory systems, which exert effects on neurons via neurotransmitters, are absent in acute slice preparations. To restore the inputs, co-cultures of slices from various brain regions have been used to study effects of neuromodulators [59].

Dissociated and randomly seeded cell cultures of electrogenic cells, such as neurons or cardiac myocytes, have been well established as biological preparations with MEAs [11, 27, 39, 60, 61] as shown in Fig. 6. Primary neurons are usually isolated from brains of embryonic or postnatal rodents, since these cells exhibit a high degree of plasticity, a characteristic that is required for developing neuronal networks in vitro [62]. The ability to record and stimulate cultured cells has provided unique experimental setups for studying the cellular activity over extended periods of time, ranging from hours to weeks [63, 64]. Moreover, patterned neuronal networks have been used to further increase the number of occupied electrodes $[65,66]$.

The organotypic tissue slice preparation is the third type of tissue preparation and provides a compromise between the longevity of the cell culture and the in vitro characteristics of the acute slice (Fig. 7). The preparation of organotypic slices is similar to that used for acute slices, except that the cultures must be rotated through liquid medium and the ambient gas during the culturing and maturation phase, which lasts for up to 4 weeks [68]. After several days, the cultures stabilize with high cell viability. Prior to recording, the culture chamber must be removed

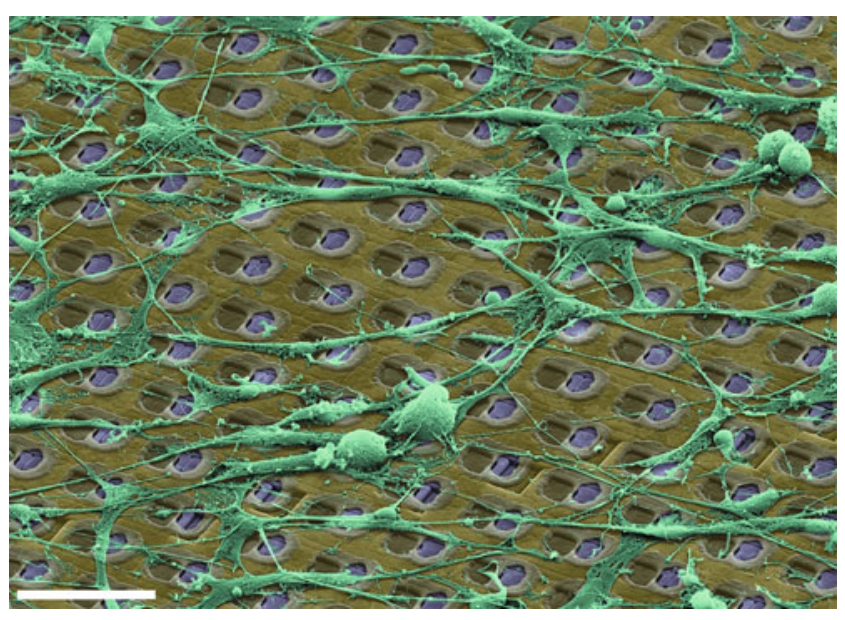

Fig. 6 A scanning electron micrograph with false coloring of chicken dorsal root ganglion neurons on the HD-MEA after 2 days of in vitro culture. Microelectrodes are colored blue and neurons are colored green; (scale bar is $30 \mu \mathrm{m}$ ) [67] from the cell culture incubator; a slow perfusion system is then used to supply carbogen gas and nutrients.

Numerous studies have involved the administration of pharmacological compounds to organotypic brain slices on MEAs for simulating pathological conditions leading to neuronal death and neurotoxicity. Examples include studies on the use of hippocampal slices cultured on MEAs and the time-course of synaptic responses and neuronal damage by using glutamate receptor agonists, $N$-methyl-D-aspartate (NMDA) and $\alpha$-amino-3-hydroxy-5-methyl-4-isoxazolepropionic acid (AMPA) [70, 71], as well as the long-term recording of long-term potentiation (LTP) after tetanic stimulation $[72,73]$.

\section{Diagnostic applications}

CMOS technology can provide electrophysiological data at unprecedented spatiotemporal resolution for several applications in basic research, as well as in applied science and industry. As an electrophysiology modality, the CMOS MEA can be considered relatively high throughput, as many data points can be collected from one sample. As already mentioned, longitudinal studies are possible, which offers the possibility to conduct chronic drug exposure studies. As will be described below, the MEA can be used for several applications: here, its potential for research in the cardiac, ocular, and nervous systems as well as its implementation for molecule detection is discussed.

\section{Cardiac system}

Arrhythmias are rhythmic disruptions of the heart, which can cause death [74]. A leading culprit for arrhythmia manifestations are arrhythmogenic prescription medications such as antibiotics, antipsychotics, antihistamines, and antiarrhythmics [75], many of which have been taken off the market because of these effects [76]. An increase in the time required for the ventricle of the heart to repolarizeknown as the "QT interval" - has been associated with ventricular tachyarrhythmia in patients [77]. The QT interval is a measure of the time between the start of the $\mathrm{Q}$ wave and the end of the $\mathrm{T}$ wave in the heart's electrical cycle. A prolonged QT interval is a risk factor for ventricular tachyarrhythmia and sudden death.

Ventricular depolarization and repolarization are the result of the complex activities of many cellular membrane ion channels, particularly the calcium and sodium channels. At the cellular level, the action potential duration (APD) of individual cardiomyocytes in vitro is the amount of time required for the cell's membrane to depolarize and repolarize back to resting membrane potential. In a simplified sense, the QT interval of the heart is a 
Fig. 7 Organotypic cultures on an MEA. a A 9-day-old organotypic co-culture of entorhinal cortex and dentate gyrus from rats and mice. b Signals recorded using the MEA following stimulation (location of stimulation is marked by asterisk). Each waveform represents the electrophysiologic activity recorded by the corresponding electrode. Dotted lines indicate locations of entorhinal cortex and dentate gyrus [69]

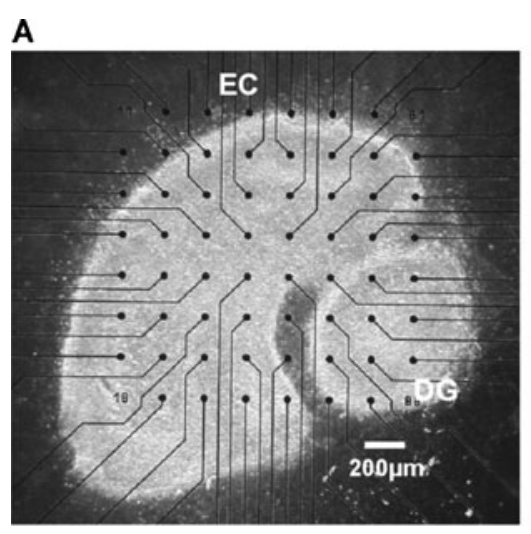

summation of the APDs of individual heart cells (the cardiomyocytes) of the heart's ventricular compartment. It is for this reason that in vitro measures of ADP in heart cells are of relevance as a screen for drug-induced disorders of the heart $[19,78,79]$.

While the above-mentioned screening methods are in use regarding the risk of QT prolongation associated with certain drugs, they are laborious and costly. An MEA, on the other hand, can provide electrophysiological information about the activity of cardiomyocytes on a finer scale, describing microcircuitry of the cardiac tissue, which may lead to a better understanding and characterization of QT prolongation [79], as shown in Fig. 8.

There have not only been several studies in which an MEA was used to record from cultured cardiomyocytes, but

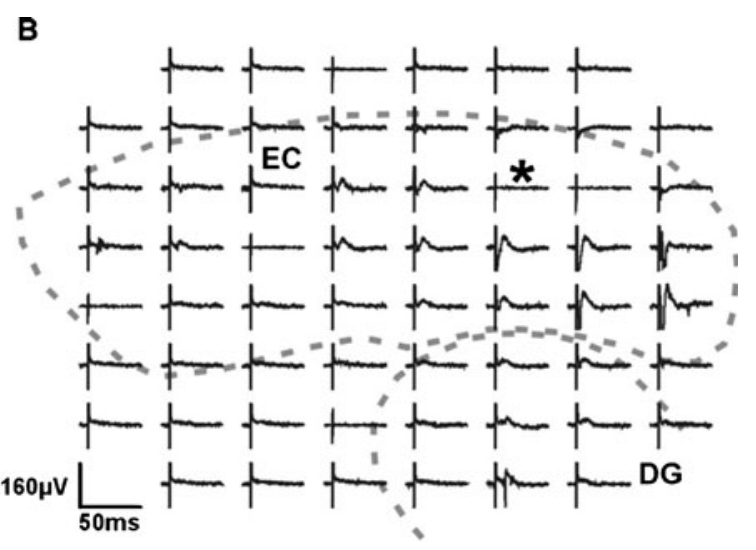

the effects of pharmacological agents have also been investigated [61,80-82]. Using a 60-electrode array, Stett et al. used antiarrhythmatic drugs quinidine and sotalol and hERG blocker E4031 to show a change in field potential of myocytes of embryonic chicken ventricles [19]. Another group used the same MEA measurement of repolarization in human embryonic stem cell-derived cells using the antiarrhythmic agent sotalol to show prolongation of the APD [83]. Meyer et al. used an MEA1060 system from Multi Channel Systems GmbH, Reutlingen, Germany recording from 60 channels, to characterize the effects of antiarrhythmic agents E4031, amiodarone, quinidine, sotalol, and verapamil on cultured embryonic chicken cardiomyocytes [78, 84]. The results for the first four drugs confirmed their QT-prolonging effects, while verapamil was
Fig. 8 Recordings of human stem cell-derived cardiomyocytes [79]. a Cardiomyocytes on an $8 \times 8$ MEA. b Corresponding signals from electrodes of the MEA. c A recording from one electrode channel, shown at a larger timescale
A

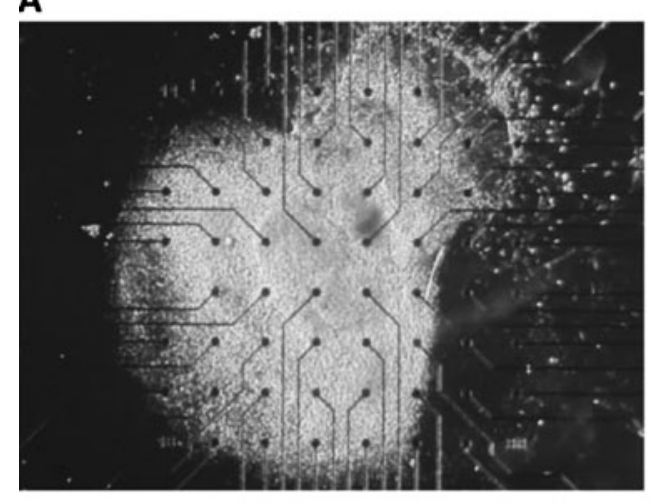

$2 \overline{00 \mu m}$

C

\#44
B

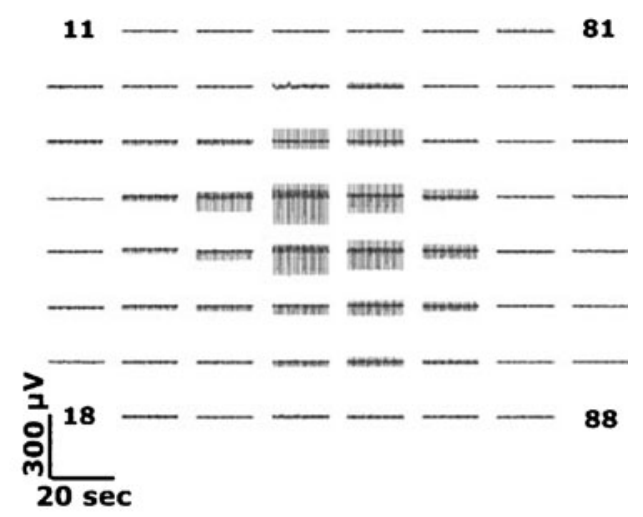

20 sec

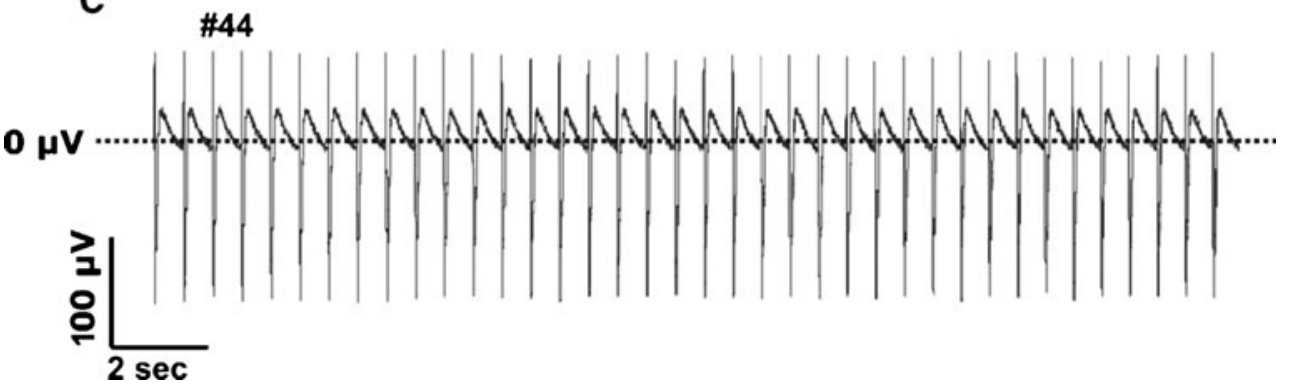


a false positive. The overall successes of these studies show the suitability of MEA systems for the characterization of antiarrhythmic agents.

Cardiac infarctions in patients have been shown to cause a phenomenon called reentry, which is a condition whereby abnormal action potential wavefronts occur in cardiac tissue; this can lead to ventricular tachycardia [85-87]. Hong et al. used optical mapping and MEAs to measure cardiac excitation waves in dissociated cardiac cell cultures. They found that high-density cultures of cardiac cells are able to sustain reentries [88].

Certain types of heart disease and rhythm disturbances cause arrhythmogenic remodeling within the heart, affecting the distribution of gap junction channels within the cardiac tissue [89]; among many effects, the cell coupling is altered [90]. Subtle changes, including action potential propagation speed, can be characterized with an MEA, which is not possible with other recording systems. Using a high-density MEA with a resolution of $17 \mu \mathrm{m}$, it was shown that the antiarrhythmic agent lidocaine modified the action potential shape as well as the signal propagation speed $[61,82]$.

\section{Retina}

The retina contains complex circuits of neurons that extract specific features from the visual input, such as changes in light intensity, color variations, and the direction of moving objects [91]. The final output of the retina is computed in the ganglion cell layer and consists of complex spatiotemporal patterns of action potentials that can be recorded with MEA technology [92]. Therefore, MEAs are ideal for accessing the electrodynamics of retinal ganglion cell populations because their cell bodies and axons are aligned in a 2D layer (Fig. 9).

Multisite extracellular recordings showed synchronized activity between retinal ganglion cells [96-98] and demonstrated that specific features of the visual scene are coded by this correlated activity across the retina [93, 99] (Fig. 9); furthermore, pharmacological studies in combination with
Fig. 9 a A patch of salamander retina placed over a multielectrode array. Ganglion cell and axon bundles are fluorescently stained with rhodamine dextrane (green) [92]. b Simultaneously recorded signals from six retinal ganglion cells as the direction of a dark bar near their receptive field centers is reversed [93]. c Responses of retinal ganglion cells to electrical stimulation on MEAs [94]. d Representation of epiretinal and subretinal implants for visual restoration [95]
A

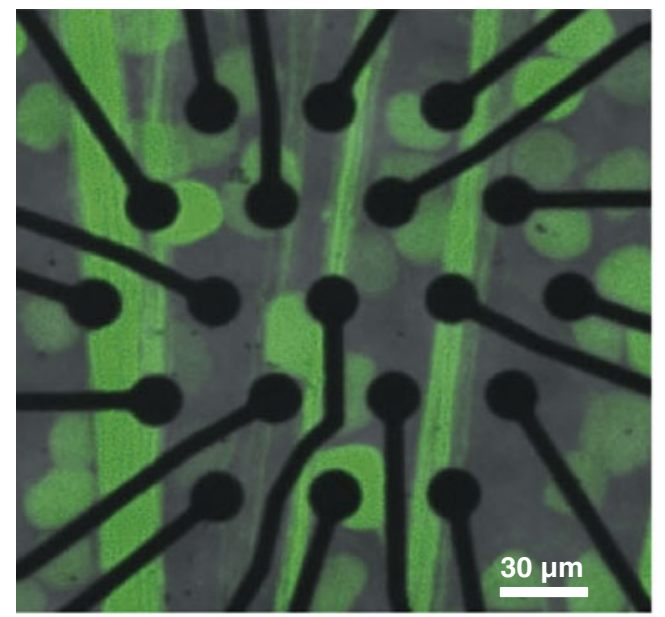

C

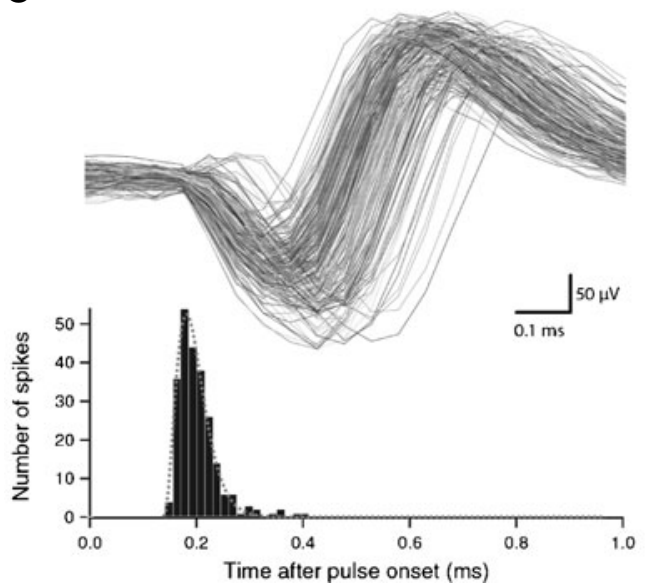

B
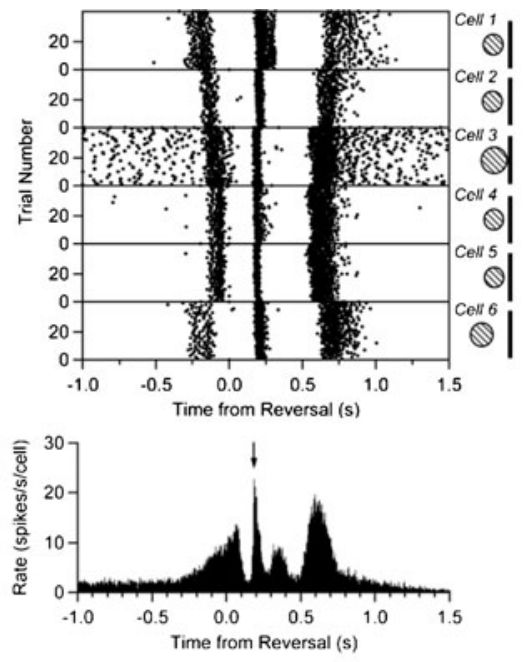

D

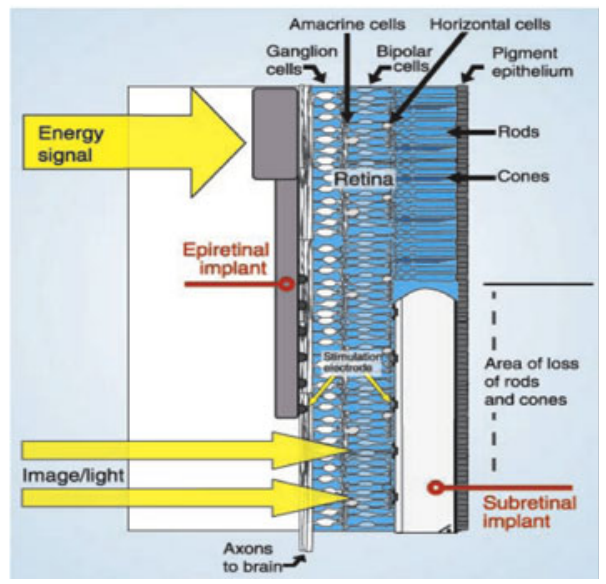


microelectrode array recordings have revealed distinct types of temporal correlations [100]. The majority of these correlations arise from electrical and chemical synapses and cause redundancy in the retinal output [101].

All these studies indicate the potential of MEAs as a fundamental tool for analyzing how retinal populations code visual input in order to communicate with higher brain centers. In addition, MEA technology provides a platform in biomedical research for developing new strategies to cure retinal diseases. Vision loss affects thousands of people around the world [102] and, in most cases, poor vision or blindness is caused by photoreceptor degeneration [103].

Multielectrode arrays have been used to analyze how electrical stimulation evokes spiking activity in retinal ganglion cells [94] (Fig. 9). Such electrical stimulations are essential for developing retinal implants for vision restoration. There are two types of retinal implants currently showing promise in clinical trials: epiretinal implants (on the retina) and subretinal implants (behind the retina), see Fig. 9d. Epiretinal implants sit on top of the retina, directly stimulating ganglia using signals sent from an external camera and power sent from an external transmitter, whereas subretinal implants sit under the retina, stimulating bipolar or ganglion cells from underneath and have provided promising results recently [104]. In the epiretinal approach, the prosthesis adjacent to the ganglion cell layer bypasses the entire retinal circuitry [95].

An alternative approach to restore photosensitivity in the retina is to express genes encoding light sensitive functions in specific retinal neuron subtypes [105]. Microelectrode arrays are a required support for this strategy because they provide a rapid and detailed analysis of the response induced by the expressed gene.

Furthermore, MEA technology is applied in developmental studies of retinal circuits [106] and to analyze the spatiotemporal properties of retinal ganglion cell receptive fields [107]; accurate description of spatiotemporal receptive fields is used to create models predicting the output of specific subtypes of retinal ganglion cells [108].

Commercial MEAs have been routinely used for retina research, nevertheless the electrode density is still too poor to access the rich amount of information coded by the different retinal ganglion cell subtypes. CMOS-based MEAs with high spatiotemporal resolution will be crucial to increase our knowledge of the retinal code and to develop efficient medical application to treat diseases of vision.

\section{Central and peripheral nervous system}

Because of its high level of complexity, our understanding of the human brain is still largely a work in progress. Elucidating the various functional circuitry of the brain has been the subject of many studies, yet the disorders that occur in the brain have not been fully characterized. It has been shown that conditions such as Alzheimer's disease (AD) and epilepsy depend on the concerted activity of neural networks. Use of cultures as surrogates for in vivo experiments is contingent on the premise that the "functional units" of the brain are distinct cellular populations [109]. As such, cultured networks of neurons can be considered the "functional unit of functional organization" in the brain [110]. The elucidation of such functional units may be expanded by using the MEA, facilitating the study of brain disorders and pathologies [69].

One of the characteristic symptoms of $\mathrm{AD}$ is the impairment of memory function [111]. Although memory mechanisms are still not well understood, two processes, long-term potentiation (LTP) and long-term depression (LTD), are thought to be opposing forces that are responsible for memory storage [112, 113]. Some light has been shed on mechanisms that cause $\mathrm{AD}$ : in the cerebral cortices of human subjects with Alzheimer's disease, amyloid beta protein $(\mathrm{Ab})$ oligomers inhibit LTP, enhance LTD, and reduce dendritic spine density in normal rodent hippocampus [114]; at the Alzheimer's Association International Conference on Alzheimer's Disease in 2009, the toxic effects of $\mathrm{Ab}$ oligomers were demonstrated by using cultured hippocampal neurons on an MEA, confirming previous data from patch clamp studies [115].

Epilepsy is the most common neurological disorder, affecting 50 million people worldwide [116]. MEA recordings were acquired from pharmacologically induced models of epileptiform activity in rat acute hippocampal slices. Hill et al. [117] used the introduction of 4-aminopyridine (4-AP) and the removal of external $\mathrm{Mg}^{2+}$ ions as in vitro models of epilepsy, as shown in Fig. 10. The effects of two anticonvulsant drugs, felbamate and phenobarbital, were assessed on these model platforms; phenobarbital was shown to completely repress all induced epileptic bursting in the slices. The characteristics typically seen in vivo, such as changes in burst duration, frequency, and amplitude, were measured in these slices [117].

For the investigation of pharmacological compounds, cultured neural networks provide a highly sensitive platform. Such a system is stable over the long-term for evoked or spontaneous electrophysiological activity [27, 61, 63]. Furthermore, sensitivities to certain compounds can be regulated by the increase or decrease of receptor expression [118]. It has also been established that cultured neural networks respond to neurotransmitters, blockers, and many pharmacological substances such that in vivo behavior of neurons can be mimicked in vitro [11, 119-122]. For example, bicuculline blocks the main inhibitory neurotransmitter $\gamma$-aminobutyric acid (GABA), causing an increased spiking frequency, as does the chloride channel antagonist 
Fig. 10 Models of epilepsy in hippocampal slices. a A hippocampal acute slice from an adult rat $(p=30)$ mounted on an MEA. Scale bar is $200 \mu \mathrm{m}$. b, c Recordings of epileptiform bursts in the slice induced by the potassium channel blocker 4-aminopyridine (4-AP) and the absence of $\mathrm{Mg}^{2+}$ in extracellular solution [117]. d, e Representative bursts from $\mathbf{b}$ and $\mathbf{c}$ [117]
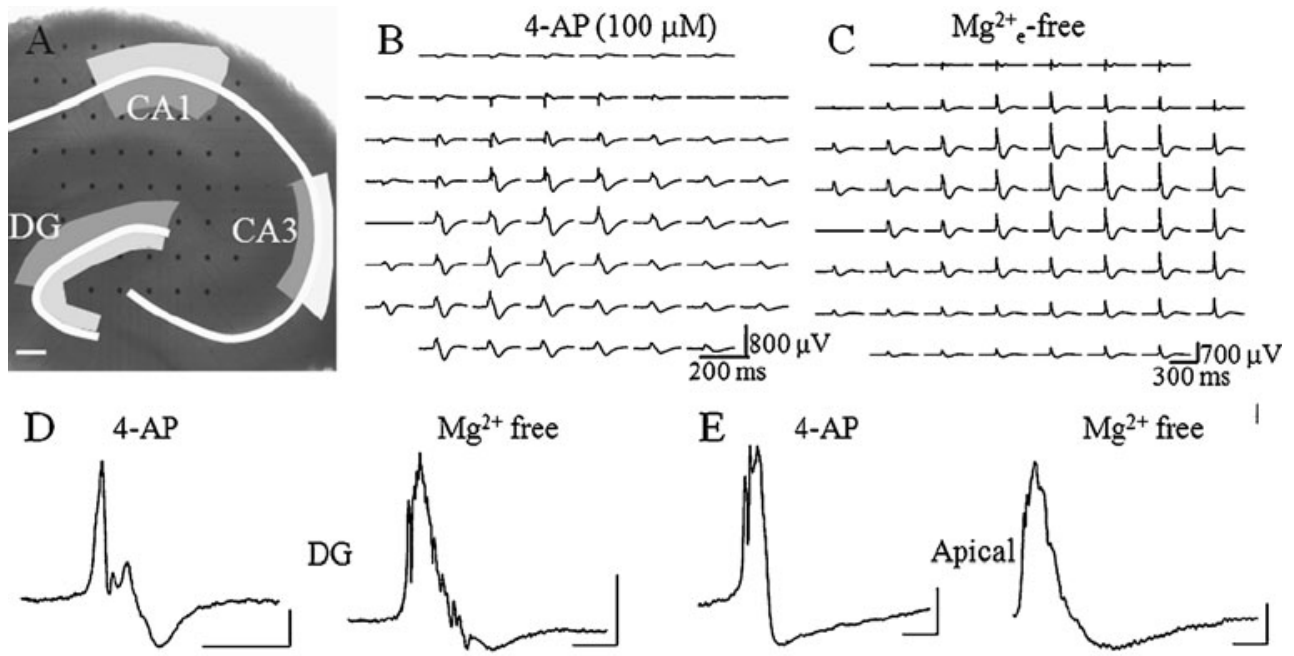

E 4-AP

$\mathrm{Mg}^{2+}$ free
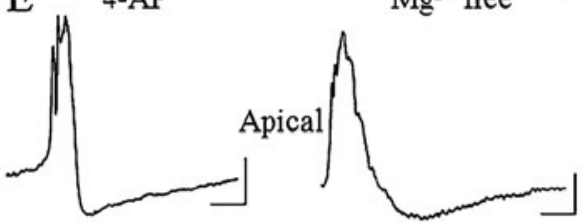

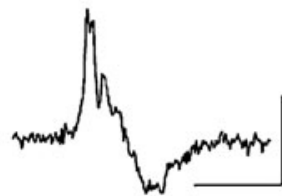

CA3
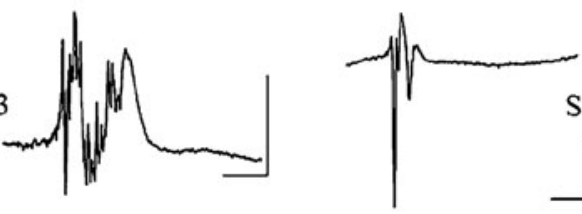

Somatic

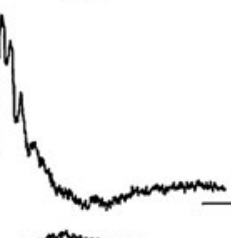

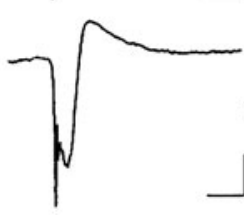

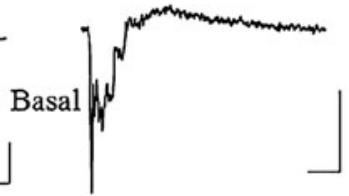

strychnine (Fig. 11). Such cultures are cultured for longterm monitoring of neuronal electrophysiological activity including development, neuronal plasticity, and axonal regeneration [123, 124], all of which may be affected by the introduction of pharmaceutical compounds.

Neurotoxicity assessment studies for new compounds, as per the guidelines of the Organization for Economic Cooperation and Development and European Community, require only in vivo neurobehavioral and neurotoxicity testing. Such testing procedures do not always accurately predict responses in humans, are costly, and require the use of numerous laboratory animals, the number of which could be reduced by the use of in vitro methods, such as described in the preceding paragraph [122]. At the 11th International Neurotoxicology Association Meeting, several in vitro models were discussed [125]. Two groups used primary dissociated cultures from mouse or rat cortex and spinal cord neuronal networks to study spontaneous and evoked neuronal activity. The effect of several pharmacological agents on spontaneous activity of cultured neural networks was assessed. A standard neurotoxicant, trimethyltin chloride, had a dose-dependent effect in mouse central nervous system (CNS), specifically the spinal cord and auditory cortex [121]. The group established five

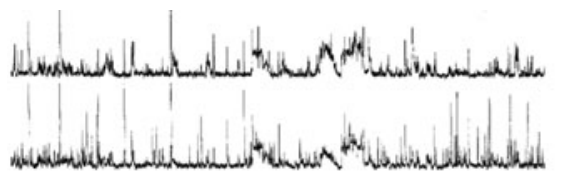

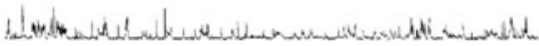

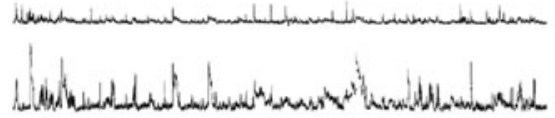

A Native
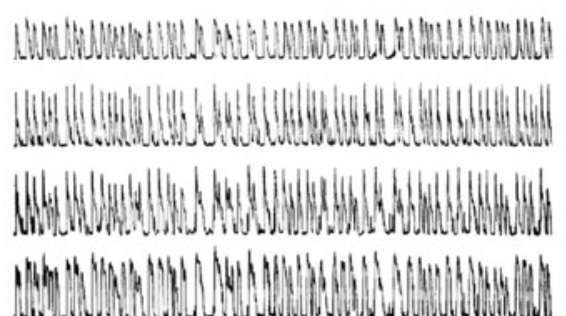

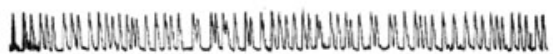

B BCC

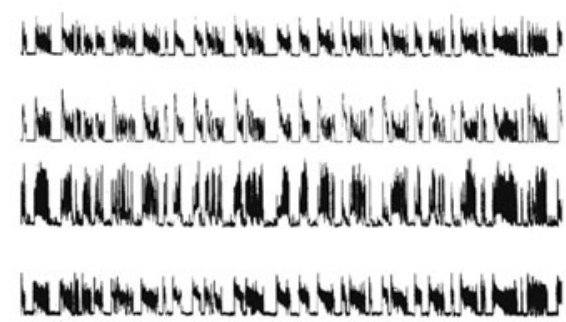

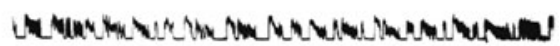
C STR

Fig. 11 Recordings from five neurons in spinal cord cultures. a Control recordings. b GABA receptor blockage by bicuculline (BCC) and $\mathbf{c}$ chloride channel antagonist strychnine (STR) [122] 
distinct drug-induced oscillatory activity states by blocking common neurotransmitter receptors. This study created "fingerprints" of characteristic responses to known and unknown substances, which can be used to evaluate responses to drugs $[122,125,126]$.

The peripheral nervous system has also been the focus of several studies. Czarnecki et al. used MEAs to study the repetitive patterns of neural activity in spinal cord that are responsible for behavior such as locomotion; they investigated neuronal networks of cells from the vertebrate spinal cord, and coupling properties were elucidated by using pharmacological methods. Bursting network activity and intrinsic activity in these cultures were modulated by 5 hydroxytryptamine (5-HT, serotonin) and the cholinergic agonist muscarine [127]. Rhythmic activity in spinal cord slices has been monitored over long durations using MEAs [128], as has been circadian rhythm activity in organotypic slices of the hypothalamic suprachiasmatic nucleus [129, 130].

\section{Biochemical sensors}

Biochemical sensors are devices that reveal information about the chemical composition of a biological system either in vitro or in vivo in real time. They make it possible to discriminate the relevant species from biologically significant background molecules and to signal the presence of a chemical or measure its concentration with an electrical signal such as current, voltage, or frequency. While some medical equipment has become more complex and more expensive, the nature of and specific applications for biochemical sensors associated with diagnosis drive them to become more compact and more economically viable.

Electrode-based electrochemical sensors constitute the oldest and most diverse group of chemical sensors, as they can be used for a large variety of applications. Potentiometric, conductometric, and voltammetric measurement techniques can be employed depending on the mechanism of the reaction to be analyzed. Potentiometric sensors operate at the equilibrium state: a potential is measured while no current is allowed to flow. The potential is thus proportional to the logarithm of the concentration of the electroactive species, as described in the Nernst equation [131]. Chemically sensitive field-effect transistors (ChemFETs) and ISFETs, in which the gate voltage is supplied by the studied chemical process, are examples of potentiometric sensors that can be used to measure $\mathrm{pH}$ [34]. Twodimensional $10 \times 10$ arrays of $\mathrm{pH}$ sensors have been constructed, which can continuously monitor sub-second $\mathrm{pH}$ changes in a solution and output visual images [132]. Conductometric sensors measure impedance (resistance or capacitance) changes between sets of electrodes with a sensing layer between them before and after exposure to analytes. Conductometric sensors are usually employed as gas sensors [131]. Capacitive sensors can also be used as gas sensors, although are more typically employed as humidity sensors [133]. Either of these types of gas sensors can be used for diagnostic purposes, such as for evaluating lung performance or measuring acetone concentration in the breath, which can indicate an exacerbation of diabetes [134], as long as they are specific and discriminating enough in real-world conditions. Notably, DNA hybridization has been detected by using arrays of capacitive sensors as well [135]. This approach will be examined in greater detail below. Finally, voltammetric sensors feature potentiostats, which enable them to perform dynamic experiments such as voltammetry (coulometry) where the voltage is modulated and the resulting current is measured, or vice versa. These electrochemical sensors are two- to three-electrode configurations and rely on charge transfer reactions between analytes and electrodes that oxidize and reduce species of interest. The resulting signals are usually linearly proportional to the concentration of the electroactive species of interest. The use of standardized reference electrodes in combination with the potentiostat allows a single half-cell reaction to be studied. Combinations of linear sweeps, pulses, and cycling of these methods within different ranges and at varying scan rates provide a wealth of information about the system being studied. Cyclic voltammetry and fast scan voltammetry are two well-known techniques, which are used to discern information about charge exchange, chemical concentrations, and adsorption of reactants [136].

CMOS chips can be designed with innumerable electrical functions, and can have electrodes or other microstructures built onto them in batch processes. These interfaces can additionally be chemically or enzymatically activated, making the possibilities of CMOS chips as biological or chemical signal sensors extensive [131].

One CMOS chip that is based on voltammetric techniques combines a multiplexed MEA of 576 platinum sensors, on-chip reference and counter electrodes, and an on-chip potentiostat, which can be used for electrochemical detection of processes such as DNA hybridization for disease detection, e.g., HIV, HSV, and malaria, and neurotransmitter detection for fundamental research or drug screening [137]. For DNA hybridization sensing, a conducting polymer and then a linking/spacing polymer are sequentially electrodeposited, and thus unlabeled, unmodified single-stranded DNA is immobilized via its naturally occurring phosphate groups onto the chip [138]. Cyclic voltammetry, in which the potential between the working and reference electrodes is ramped up and down, is applied before and after the introduction of sample ("target") DNA, and hybridization is measured as a diminution in the 
current. The MEA structure creates excellent opportunities in terms of diagnosis in that different DNA can be deposited on different electrodes, making it possible to test for many diseases or strains of a disease with a single sample and in a single measurement cycle [137, 138].

Another chip, which utilizes a capacitance variation upon DNA hybridization, further demonstrates the versatility of CMOS to accomplish the same goal (Fig. 12). Thiolated, single-stranded DNA covalently bonds to gold electrodes in one chamber of a dual-chambered $8 \times 16$ array of interdigitated electrodes [139]. Sample DNA is then introduced into both chambers (bare electrodes and immobilized target DNA) and the changes in capacitance are measured by converting changes in voltage to changes in frequency. This is accomplished by exciting the electrodes with current pulses and measuring their responses, which are voltage changes whose waveform time constant is dominated by the capacitive component of the interface impedance.

Yet another approach functions both as a hybridization and polymerization sensor, and could therefore be used to sequence DNA [140]. Probe DNA is immobilized over a polymer layer by spotting, and target DNA of a different length is then introduced. The shorter strand is then extended via computer-controlled deoxynucleotide triphosphate (dNTP) release, and at each incorporation of a nucleotide, a proton is released which diffuses quickly away. This transient current is the measured signal [141].

For all of these CMOS approaches, redundant measurements for improved statistics can be performed, since many different electrodes can easily be prepared with the same DNA. Additionally, chips can be activated with many kinds of DNA specific to a subset of patients based on their

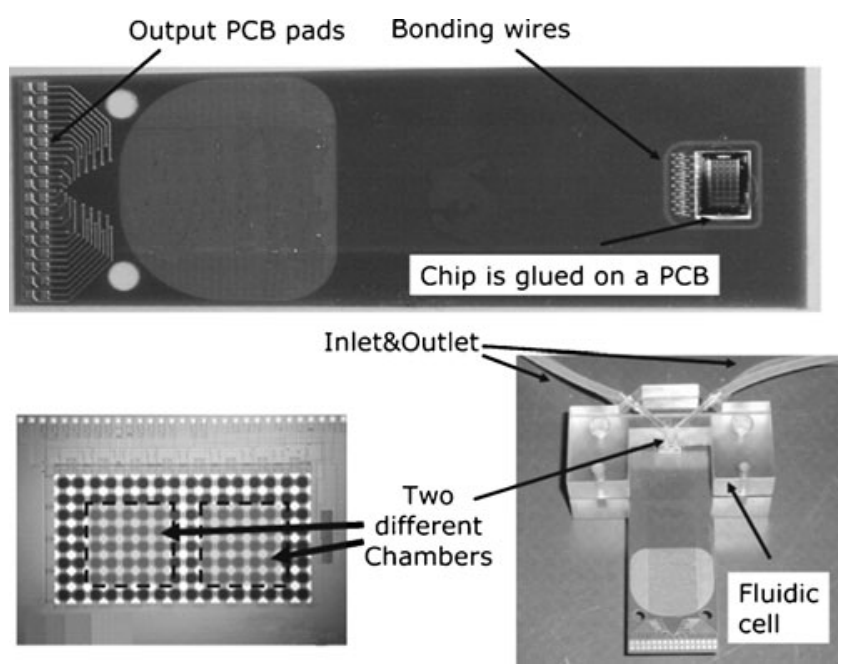

Fig. 12 Microsensor MEA chip with $8 \times 16$ array of capacitive sensors shown with printed circuit board (PCB) and fluidic cell [139] susceptibility or exposure so that a single measurement could test for many diseases and parasites.

Several of these chips can also be used to detect electroactive neurotransmitters, such as dopamine, norepinephrine, epinephrine, and serotonin [142], or can be functionalized with enzymes for the detection of neurotransmitters that are oxidized by those enzymes and produce the electroactive by-product hydrogen peroxide (i.e., glutamate, acetylcholine, choline, glucose-each oxidized by its respective oxidase) for in vitro and in vivo applications [143]. Although noble metal electrodes have rarely been used for detection via oxidation of electroactive neurotransmitters (carbon fiber electrodes were most often used), they are now becoming more popular as they can be coated with polymers which extend their potential [144]. With metal electrodes, techniques such as fast scan cyclic voltammetry, in which entire measurement cycles can be made in milliseconds, and differential pulse voltammetry can be used to identify chemicals of interest and monitor their concentrations. It is important to have very sensitive and selective techniques because many different chemicals are present in the brain, which interact and interfere with each other [30]. The electrochemical techniques are very useful for the application, but other methods are needed for non-electroactive neurotransmitters. For these, enzymes can be affixed to only one of two adjacent electrodes and differential measurements can be performed. Selective membranes (permeable only to certain molecules) can also be used as long as they do not slow down reaction times to unacceptable levels [30].

There also exist a number of different other nonelectrochemical mechanisms, which can be employed for the recognition of the pertinent molecule(s) as well as for signal transduction, which have evolved and become significantly more sophisticated over the years. With regard to sensing methods that can effectively be realized in CMOS, mass-sensitive, thermal, or optical detection means have been developed, which can employ either equilibrium or kinetic selectivity measures [145]. Mass-sensitive sensors, e.g., employ techniques such as the deflection or resonance of very small stiff cantilevers upon mass accretion. These can be produced on CMOS as microelectromechanical or nanoelectromechanical structures (MEMS or NEMS), and detect changes of mass due to adsorption, deposition, evaporation, or erosion or as a result of an affinity binding reaction [145]. Commercially available CMOS image sensors have become popular in digital cameras, although more sensitive devices than these are required for the very small signals associated with biochemical sensing. CMOS-photodiode microluminometers for the detection of chemi- or bioluminescent signals from as few as 5,000 fluorescently labeled cells (pA currents) have been realized [146], as have arrays with 
high frame rate imagers with timing resolutions exceeding $150 \mathrm{ps}$ which are able to resolve fluorescent lifetimes [147].

\section{Conclusion}

CMOS has been shown to be a powerful technology for the construction of microdevices for biomedical research and pharmaceutical screening. The planar MEAs described are capable of measuring electrophysiological activity of multiple cells simultaneously, providing information about the activity of individual cells at high spatiotemporal resolution. Moreover, integrated electrode arrays are suitable tools to perform highly localized and distributed electrochemical sensing in biological preparations.

In the future, systems with an increased number of recording and stimulation electrodes will be developed. The use of CMOS technologies with small minimum feature size (i.e., $0.35-\mu \mathrm{m}$ or $0.18-\mu \mathrm{m}$ technology) will accelerate system innovation, allowing also the integration of more advanced analog front ends and digital interfaces with even smaller size and lower power consumption, which are needed to reduce heating that could damage the on-chip cultured cells. At the same time, such complex systems generate huge amounts of data, requiring advanced data sorting and compression. The development of novel algorithms and filters will allow data acquisition in real time. Finally, MEAs will be integrated with different sensor types, such as chemical sensors, so that even more comprehensive studies can be performed, such as the observation of cell metabolism, monitoring of neurotransmitters, the study of cell location and of tissue morphology.

The devices described have important implications in biomedical research, opening up new avenues for understanding human disease. Vastly complex organs, such as neuronal ensembles or cardiac tissue, can be investigated at a greater level of detail and pathologic mechanisms can be mimicked, studied, and perturbed by using pharmacologic or electrophysiological stimuli. The authors of this review anticipate that MEAs will have a substantial impact on diagnostics and pharmacology in the near future. While MEAs have not yet been formally accepted as a standard method for such diagnostics, it seems highly probable that these devices will play an increasingly vital role in the field as physiologic testing platforms for pharmacology require ever higher throughput dynamics and capabilities.

\section{References}

1. Neher E, Sakmann B (1976) Single-channel currents recorded from membrane of denervated frog muscle fibres. Nature 260 (5554):799-802
2. Cole KS (1949) Dynamic electrical characteristics of the squid axon membrane. Arch Sci Physiol 3(2):253-258

3. Farre $\mathrm{C}$ et al (2008) Ion channel screening-automated patch clamp on the rise. Drug Discov Today Tech 5(1):23-28

4. Fromherz P (2002) Electrical interfacing of nerve cells and semiconductor chips. Chemphyschem 3(3):276-284

5. Wise KD et al (2004) Wireless implantable microsystems: highdensity electronic interfaces to the nervous system. Proc IEEE 92 (1):76-97

6. Olsson R III et al (2005) Band-tunable and multiplexed integrated circuits for simultaneous recording and stimulation with microelectrode arrays. IEEE Trans Biomed Eng 52 (7):1303-1311

7. Kleber AG, Rudy Y (2004) Basic mechanisms of cardiac impulse propagation and associated arrhythmias. Physiol Rev 84(2):431-488

8. Rutten WL (2002) Selective electrical interfaces with the nervous system. Annu Rev Biomed Eng 4:407-452

9. Jimbo Y, Robinson HP (2000) Propagation of spontaneous synchronized activity in cortical slice cultures recorded by planar electrode arrays. Bioelectrochemistry 51(2):107-115

10. van Pelt $J$ et al (2004) Long-term characterization of firing dynamics of spontaneous bursts in cultured neural networks. IEEE Trans Biomed Eng 51(11):2051-2062

11. Morefield SI et al (2000) Drug evaluations using neuronal networks cultured on microelectrode arrays. Biosens Bioelectron 15(7-8):383-396

12. Pancrazio JJ et al (2003) A portable microelectrode array recording system incorporating cultured neuronal networks for neurotoxin detection. Biosens Bioelectron 18(11):1339-1347

13. DeBusschere BD, Kovacs GTA (2001) Portable cell-based biosensor system using integrated CMOS cell-cartridges. Biosens Bioelectron 16(7-8):543-556

14. Taketani M (2006) Advances in network electrophysiology: using multi-electrode arrays. Springer, New York

15. Voelker M, Fromherz P (2005) Signal transmission from individual mammalian nerve cell to field-effect transistor. Small 1(2):206-210

16. Jenkner M, Muller B, Fromherz P (2001) Interfacing a silicon chip to pairs of snail neurons connected by electrical synapses. Biol Cybern 84(4):239-249

17. Maher MP et al (1999) The neurochip: a new multielectrode device for stimulating and recording from cultured neurons. $\mathrm{J}$ Neurosci Methods 87(1):45-56

18. Martinoia S et al (2004) Cultured neurons coupled to microelectrode arrays: circuit models, simulations and experimental data. IEEE Trans Biomed Eng 51(5):859-864

19. Stett A et al (2003) Biological application of microelectrode arrays in drug discovery and basic research. Anal Bioanal Chem 377(3):486-495

20. Heuschkel MO et al (2002) A three-dimensional multi-electrode array for multi-site stimulation and recording in acute brain slices. J Neurosci Methods 114(2):135-148

21. Jimbo $Y$ et al (2003) A system for MEA-based multisite stimulation. IEEE Trans Biomed Eng 50(2):241-248

22 . Baker BJ et al (2005) Imaging brain activity with voltage- and calcium-sensitive dyes. Cell Mol Neurobiol 25(2):245-282

23. Peterlin ZA et al (2000) Optical probing of neuronal circuits with calcium indicators. Proc Natl Acad Sci U S A 97(7):36193624

24. Obaid AL et al (2004) Novel naphthylstyryl-pyridium potentiometric dyes offer advantages for neural network analysis. J Neurosci Methods 134(2):179-190

25. Heer $F$ et al (2007) Single-chip microelectronic system to interface with living cells. Biosens Bioelectron 22(11):25462553 
26. Grumet AE, Wyatt JL Jr, Rizzo JF 3rd (2000) Multi-electrode stimulation and recording in the isolated retina. J Neurosci Methods 101(1):31-42

27. Wagenaar DA et al (2005) Controlling bursting in cortical cultures with closed-loop multi-electrode stimulation. J Neurosci 25(3):680-688

28. Hafizovic S, Heer F, Ugniwenko T, Frey U, Blau A, Ziegler C, Hierlemann A (2007) CMOS-based microelectrode arrays for interacting with neurons. J. Neurosci Methods 164:93-106

29. Heer $F$ et al (2007) Using microelectronics technology to communicate with living cells. Conf Proc IEEE Eng Med Biol Soc 2007:6082-6085

30. Robinson DL et al (2008) Monitoring rapid chemical communication in the brain. Chem Rev 108(7):2554-2584

31. Thomas CA Jr et al (1972) A miniature microelectrode array to monitor the bioelectric activity of cultured cells. Exp Cell Res 74 (1):61-66

32. Gross GW et al (1977) A new fixed-array multi-microelectrode system designed for long-term monitoring of extracellular single unit neuronal activity in vitro. Neurosci Lett 6(2-3):101-105

33. Pine J (1980) Recording action potentials from cultured neurons with extracellular microcircuit electrodes. J Neurosci Methods 2 (1):19-31

34. Bergveld P (2003) Thirty years of ISFETOLOGY - What happened in the past 30 years and what may happen in the next 30 years. Sens Actuators B Chem 88(1):1-20

35. Bergveld P (1970) Development of an ion-sensitive solid-state device for neurophysiological measurements. IEEE Trans Biomed Eng 17(1):70-71

36. Bergveld P (1972) Development, operation, and application of the ion-sensitive field-effect transistor as a tool for electrophysiology. IEEE Trans Biomed Eng 19(5):342-351

37. Jobling DT, Smith JG, Wheal HV (1981) Active microelectrode array to record from the mammalian central nervous system in vitro. Med Biol Eng Comput 19(5):553-560

38. Hutzler $M$ et al (2006) High-resolution multitransistor array recording of electrical field potentials in cultured brain slices. J Neurophysiol 96(3):1638-1645

39. Berdondini L et al (2005) High-density electrode array for imaging in vitro electrophysiological activity. Biosens Bioelectron 21(1):167-174

40. Eversmann B et al (2003) A $128 \times 128$ CMOS biosensor array for extracellular recording of neural activity. IEEE J Solid-State Circuits 38(12):2306-2317

41. Frey U et al (2007) An 11 k-electrode 126-channel high-density microelectrode array to interact with electrogenic cells. In: Solidstate circuits conference, San Francisco, 2007 (ISSCC 2007). Digest of technical papers. IEEE International

42. Frey U et al (2009) Microelectronic system for high-resolution mapping of extracellular electric fields applied to brain slices. Biosens Bioelectron 24(7):2191-2198

43. Lambacher A et al (2004) Electrical imaging of neuronal activity by multi-transistor-array (MTA) recording at $7.8 \mu \mathrm{m}$ resolution. Appl Phys A: Mater Sci Process 79(7):1607-1611

44. Imfeld $\mathrm{K}$ et al (2007) High-resolution MEA platform for in-vitro electrogenic cell networks imaging. Conf Proc IEEE Eng Med Biol Soc 2007:6086-6089

45. Maccione A et al (2009) Imaging extracellular neuronal signaling on high resolution microelectrode arrays (MEAs). Hippocampal cultures coupled with a high resolution neuroelectronic interface. In: 4th international IEEE/EMBS conference on neural engineering, NER '09, Antalya, 2009

46. Berdondini L et al (2009) Active pixel sensor array for high spatio-temporal resolution electrophysiological recordings from single cell to large scale neuronal networks. Lab Chip 9 (18):2644-2651
47. Kovacs GTA (2003) Electronic sensors with living cellular components. Proc IEEE 91(6):915-929

48. Sodagar AM, Wise KD, Najafi K (2007) A fully integrated mixed-signal neural processor for implantable multichannel cortical recording. IEEE Trans Biomed Eng 54(6 Pt 1):10751088

49. Wise KD et al (2008) Microelectrodes, microelectronics, and implantable neural microsystems. Proc IEEE 96(7):1184-1202

50. Heer F et al (2006) CMOS microelectrode array for bidirectional interaction with neuronal networks. IEEE J Solid-State Circuits 41(7):1620-1629

51. Hafizovic S et al (2007) A CMOS-based microelectrode array for interaction with neuronal cultures. J Neurosci Methods 164 (1):93-106

52. Livi $\mathrm{P}$ et al (2010) Compact voltage and current stimulation buffer for high-density microelectrode arrays. In: Solid-state circuits conference, San Francisco, 2010 (ISSCC 2010). Digest of technical papers. IEEE International

53. Greve F et al (2007) Molecular design and characterization of the neuron-microelectrode array interface. Biomaterials 28 (35):5246-5258

54. Soussou WV et al (2007) Neuronal network morphology and electrophysiology of hippocampal neurons cultured on surfacetreated multielectrode arrays. IEEE Trans Biomed Eng 54 (7):1309-1320

55. Egert U et al (1998) A novel organotypic long-term culture of the rat hippocampus on substrate-integrated multielectrode arrays. Brain Res Brain Res Protoc 2(4):229-242

56. Frey U et al (2009) Depth recording capabilities of planar highdensity microelectrode arrays. Proceedings of the 4th international IEEE EMBS conference on neural engineering, Antalya, Turkey, pp 207-210

57. Frey U et al (2009) Unpublished results

58. Lossi L et al (2009) Cell death and proliferation in acute slices and organotypic cultures of mammalian CNS. Prog Neurobiol 88 (4):221-245

59. Hofmann F et al (2004) Functional re-establishment of the perforant pathway in organotypic co-cultures on microelectrode arrays. Brain Res 1017(1-2):184-196

60. Potter SM (2001) Distributed processing in cultured neuronal networks. Prog Brain Res 130:49-62

61. Frey U et al (2007) Cell recordings with a CMOS high-density microelectrode array. Conf Proc IEEE Eng Med Biol Soc 2007:167-170

62. Alvarez VA, Sabatini BL (2007) Anatomical and physiological plasticity of dendritic spines. Annu Rev Neurosci 30:79-97

63. Potter SM, DeMarse TB (2001) A new approach to neural cell culture for long-term studies. J Neurosci Methods 110(1-2):17-24

64. Brewer GJ et al (2008) NbActiv4 medium improvement to Neurobasal/B27 increases neuron synapse densities and network spike rates on multielectrode arrays. J Neurosci Methods 170 (2): $181-187$

65. Franks W et al (2007) Patterned cell adhesion by self-assembled structures for use with a CMOS cell-based biosensor. Biosens Bioelectron 22(7):1426-1433

66. Jungblut $M$ et al (2009) Triangular neuronal networks on microelectrode arrays: an approach to improve the properties of low-density networks for extracellular recording. Biomed Microdevices 11(6): 1269-1278

67. Frey U (2007) High-density neural interface and microhotplate gas sensor in CMOS technology, in Physical Electronics Laboratory, PEL. 2007. ETH Zurich, Zurich, Switzerland

68. Gahwiler BH et al (1997) Organotypic slice cultures: a technique has come of age. Trends Neurosci 20(10):471-477

69. Hofmann F, Bading H (2006) Long term recordings with microelectrode arrays: studies of transcription-dependent neuro- 
nal plasticity and axonal regeneration. J Physiol Paris 99(23): $125-132$

70. Krause $\mathrm{M}$ et al (2008) Altered dendritic integration in hippocampal granule cells of spatial learning-impaired aged rats. J Neurophysiol 99(6):2769-2778

71. Shimono K et al (2002) Chronic multichannel recordings from organotypic hippocampal slice cultures: protection from excitotoxic effects of NMDA by non-competitive NMDA antagonists. J Neurosci Methods 120(2):193-202

72. Jimbo Y, Tateno T, Robinson HP (1999) Simultaneous induction of pathway-specific potentiation and depression in networks of cortical neurons. Biophys J 76(2):670-678

73. Shimono K et al (2000) Origins and distribution of cholinergically induced beta rhythms in hippocampal slices. J Neurosci 20 (22):8462-8473

74. Goldberger JJ et al (2008) American Heart Association/ American College of Cardiology Foundation/Heart Rhythm Society scientific statement on noninvasive risk stratification techniques for identifying patients at risk for sudden cardiac death: a scientific statement from the American Heart Association Council on Clinical Cardiology Committee on Electrocardiography and Arrhythmias and Council on Epidemiology and Prevention. Heart Rhythm 5(10):e1-e21

75. Roden DM (2006) Long QT syndrome: reduced repolarization reserve and the genetic link. J Intern Med 259(1):59-69

76. Piccini JP et al (2009) Current challenges in the evaluation of cardiac safety during drug development: translational medicine meets the Critical Path Initiative. Am Heart J 158 (3):317-326

77. Bode G, Olejniczak K (2002) ICH topic: the draft ICH S7B step 2: note for guidance on safety pharmacology studies for human pharmaceuticals. Fundam Clin Pharmacol 16(2):105-118

78. Meyer $\mathrm{T}$ et al (2004) Micro-electrode arrays in cardiac safety pharmacology: a novel tool to study QT interval prolongation. Drug Saf 27(11):763-772

79. Reppel $\mathrm{M}$ et al (2005) The electrocardiogram of human embryonic stem cell-derived cardiomyocytes. J Electrocardiol 38(4 Suppl):166-170

80. Feld Y et al (2002) Electrophysiological modulation of cardiomyocytic tissue by transfected fibroblasts expressing potassium channels: a novel strategy to manipulate excitability. Circulation 105(4):522-529

81. Marom S, Shahaf G (2002) Development, learning and memory in large random networks of cortical neurons: lessons beyond anatomy. Q Rev Biophys 35(1):63-87

82. Sanchez-Bustamante CD et al (2008) Modulation of cardiomyocyte electrical properties using regulated bone morphogenetic protein-2 expression. Tissue Eng A 14(12):1969-1988

83. Reppel M et al (2004) Microelectrode arrays: a new tool to measure embryonic heart activity. J Electrocardiol 37(Supplement 1):104 109

84. Meyer T et al (2004) QT-screen: high-throughput cardiac safety pharmacology by extracellular electrophysiology on primary cardiac myocytes. Assay Drug Dev Technol 2 (5):507-514

85. Chang MG et al (2009) Spiral waves and reentry dynamics in an in vitro model of the healed infarct border zone. Circ Res 105 (11): 1062

86. Davidenko JM et al (1992) Stationary and drifting spiral waves of excitation in isolated cardiac muscle. Nature 355 (6358):349-351

87. Winfree A (1989) Electrical instability in cardiac muscle: phase singularities and rotors. J Theor Biol 138(3):353-405

88. Hong JH et al (2008) Spiral reentry waves in confluent layer of HL-1 cardiomyocyte cell lines. Biochem Biophys Res Commun 377(4):1269-1273
89. Kanno S, Saffitz JE (2001) The role of myocardial gap junctions in electrical conduction and arrhythmogenesis. Cardiovasc Pathol 10(4):169-177

90. Nattel S et al (2007) Arrhythmogenic ion-channel remodeling in the heart: heart failure, myocardial infarction, and atrial fibrillation. Physiol Rev 87(2):425

91. Wassle H (2004) Parallel processing in the mammalian retina. Nat Rev Neurosci 5(10):747-757

92. Segev R et al (2004) Recording spikes from a large fraction of the ganglion cells in a retinal patch. Nat Neurosci 7(10):1154 1161

93. Schwartz G et al (2007) Synchronized firing among retinal ganglion cells signals motion reversal. Neuron 55(6):958-969

94. Sekirnjak C et al (2008) High-resolution electrical stimulation of primate retina for epiretinal implant design. J Neurosci 28 (17):4446-4456

95. Zrenner E (2002) Will retinal implants restore vision? Science 295(5557):1022-1025

96. Meister M, Lagnado L, Baylor DA (1995) Concerted signaling by retinal ganglion cells. Science 270(5239):1207-1210

97. Schnitzer MJ, Meister M (2003) Multineuronal firing patterns in the signal from eye to brain. Neuron 37(3):499-511

98. Shlens J, Rieke F, Chichilnisky E (2008) Synchronized firing in the retina. Curr Opin Neurobiol 18(4):396-402

99. Ackert JM et al (2006) Light-induced changes in spike synchronization between coupled ON direction selective ganglion cells in the mammalian retina. J Neurosci 26(16):4206-4215

100. Brivanlou IH, Warland DK, Meister M (1998) Mechanisms of concerted firing among retinal ganglion cells. Neuron 20(3):527-539

101. Puchalla JL et al (2005) Redundancy in the population code of the retina. Neuron 46(3):493-504

102. Chader GJ, Weiland J, Humayun MS (2009) Artificial vision: needs, functioning, and testing of a retinal electronic prosthesis. Prog Brain Res 175:317-332

103. Rattner A, Sun H, Nathans J (1999) Molecular genetics of human retinal disease. Annu Rev Genet 33:89-131

104. Zrenner E et al (2009) Subretinal microelectrode arrays allow blind retinitis pigmentosa patients to recognize letters and combine them to words. Proceedings of the 2009 2nd international conference on biomedical engineering and informatics (BMEI), p 4

105. Lagali PS et al (2008) Light-activated channels targeted to ON bipolar cells restore visual function in retinal degeneration. Nat Neurosci 11(6):667-675

106. Elstrott $\mathrm{J}$ et al (2008) Direction selectivity in the retina is established independent of visual experience and cholinergic retinal waves. Neuron 58(4):499-506

107. Devries SH, Baylor DA (1997) Mosaic arrangement of ganglion cell receptive fields in rabbit retina. J Neurophysiol 78(4):2048-2060

108. Chichilnisky EJ (2001) A simple white noise analysis of neuronal light responses. Network 12(2):199-213

109. Edelman GM (1987) Neural Darwinism: the theory of neuronal group selection. Oxford University Press, Oxford

110. MacGregor RJ (1987) Neural and brain modeling. Academic, New York

111. Rowan MJ et al (2005) Synaptic plasticity disruption by amyloid beta protein: modulation by potential Alzheimer's disease modifying therapies. Biochem Soc Trans 33(Pt 4):563-567

112. Bliss TV, Collingridge GL (1993) A synaptic model of memory: long-term potentiation in the hippocampus. Nature 361 (6407):31-39

113. Lynch MA (2004) Long-term potentiation and memory. Physiol Rev 84(1):87-136

114. Shankar GM et al (2008) Amyloid-beta protein dimers isolated directly from Alzheimer's brains impair synaptic plasticity and memory. Nat Med 14(8):837-842 
115. Varghese K et al (2009) Microelectrode arrays and Alzheimer's disease: a novel in vitro investigative paradigm. Alzheimers Dement 5(4, Supplement 1):P428-P428

116. Scott RA, Lhatoo SD, Sander JWAS (2001) The treatment of epilepsy in developing countries: where do we go from here? Bull World Health Organ 79:344-351

117. Hill AJ et al (2009) Development of multi-electrode array screening for anticonvulsants in acute rat brain slices. J Neurosci methods

118. Gross GW et al (1995) The use of neuronal networks on multielectrode arrays as biosensors. Biosens Bioelectron 10(67):553-567

119. Streit J (1993) Regular oscillations of synaptic activity in spinal networks in vitro. J Neurophysiol 70(3):871

120. Gross GW et al (1997) Odor, drug and toxin analysis with neuronal networks in vitro: extracellular array recording of network responses. Biosens Bioelectron 12(5):373-393

121. Gramowski A, Schiffmann D, Gross G (2000) Quantification of acute neurotoxic effects of trimethyltin using neuronal networks cultured on microelectrode arrays. Neurotoxicology 21(3):331

122. Gramowski A et al (2004) Substance identification by quantitative characterization of oscillatory activity in murine spinal cord networks on microelectrode arrays. Eur J Neurosci 19(10):2815-2825

123. Shahaf G, Marom S (2001) Learning in networks of cortical neurons. J Neurosci 21(22):8782-8788

124. Arnold FJ et al (2005) Microelectrode array recordings of cultured hippocampal networks reveal a simple model for transcription and protein synthesis-dependent plasticity. J Physiol 564(Pt 1):3-19

125. Bal-Price AK et al (2008) Application of in vitro neurotoxicity testing for regulatory purposes: symposium III summary and research needs. Neurotoxicology 29(3):520-531

126. Gross GW, Schwalm FU (1994) A closed flow chamber for longterm multichannel recording and optical monitoring. J Neurosci Methods 52(1):73-85

127. Czarnecki A, Magloire V, Streit J (2008) Local oscillations of spiking activity in organotypic spinal cord slice cultures. Eur J Neurosci 27(8):2076-2088

128. Tscherter A et al (2001) Spatiotemporal characterization of rhythmic activity in rat spinal cord slice cultures. Eur J Neurosci 14(2):179-190

129. Honma S, Honma K (2003) The biological clock: Ca2+ links the pendulum to the hands. Trends Neurosci 26(12):650-653

130. Reppert SM, Weaver DR (2001) Molecular analysis of mammalian circadian rhythms. Annu Rev Physiol 63:647-676
131. Hierlemann A, Baltes H (2003) CMOS-based chemical microsensors. Analyst 128(1):15-28

132. Sawada $\mathrm{K}$ et al (2005) A novel fused sensor for photo-and ionsensing. Sens Actuators B Chem 106(2):614-618

133. Joo S, Brown RB (2008) Chemical sensors with integrated electronics. Chem Rev 108(2):638-651

134. Ryabtsev $\mathrm{S}$ et al (1999) Application of semiconductor gas sensors for medical diagnostics. Sens Actuators B 59(1):26-29

135. Stagni C et al (2007) A fully electronic label-free DNA sensor chip. IEEE Sens J 7(4):577-585

136. Bard AJ, Faulkner LR (2001) Electrochemical methods: fundamentals and applications, 2nd edn. Wiley, New York

137. Heer F, Keller M, Yu G, Janata J, Josowicz M, Hierlemann A (2008) CMOS electro-chemical DNA-detection array with onchip ADC. In: Proceedings of the IEEE International solid-state circuits conference (ISSCC), San Francisco, 2008

138. Aiyejorun T, Thompson L, Kowalik J, Josowicz M, Janata J (2005) Control of chloride ion exchange by DNA hybridization at polypyrrole electrode. Perspec Bioanal 1:331-344

139. Stagni $C$ et al (2006) CMOS DNA sensor array with integrated A/D conversion based on label-free capacitance measurement. IEEE J Solid-State Circuits 41(12):2956-2964

140. Anderson E, Daniels J, Yu H, Lee T, Pourmand N (2008) A label-free CMOS DNA microarray based on charge sensing. In: Proceedings of the IEEE instrumentation and measurement technology conference, Vancouver Island, 12-15 May, 2008

141. Pourmand $N$ et al (2006) Direct electrical detection of DNA synthesis. Proc Natl Acad Sci U S A 103(17):6466

142. Borland L, Michael A (2007) An introduction to electrochemical methods in neuroscience, CRC, Boca Raton

143. Frey O (2010) Biosensor microprobe array for in vivo monitoring of neurotransmitters. EPF Lausanne, Switzerland

144. Laubender E et al (2009) Polymer composite coated multifiber array microelectrodes as multifunctional voltammetric microsensor for the detection of dopamine

145. Janata J (2008) Principles of chemical sensors, 2nd edn. Springer, Dordrecht

146. Bolton EK et al (2002) Integrated CMOS photodetectors and signal processing for very low-level chemical sensing with the bioluminescent bioreporter integrated circuit. Sens Actuators B Chem 85(1-2):179-185

147. Huang TCD, Sorgenfrei S et al (2009) A 0.18- $\mu \mathrm{m}$ CMOS array sensor for integrated time-resolved fluorescence detection. IEEE J Solid-State Circuits 44(5):1644-1654 